\title{
Method for data processing in application to ohmic contacts
}

\author{
A.E. Belyaev ${ }^{1}$, N.S. Boltovets ${ }^{2}$, R.V. Konakova ${ }^{1}$, V.M. Kovtonjuk ${ }^{1,2}$, Ya.Ya. Kudryk ${ }^{1 *}$, V.V. Shynkarenko ${ }^{1}$, \\ M.M. Dub ${ }^{1}$, P.O. Saj ${ }^{1}$, S.V. Novitskii ${ }^{3}$
}

${ }^{I}$ V. Lashkaryov Institute of Semiconductor Physics, NAS of Ukraine,

41, prospect Nauky, 03680 Kyiv, Ukraine

${ }^{2}$ State Enterprise "Research Institute "Orion",

8a, Eugene Pottier str., 03057 Kyiv, Ukraine

${ }^{3}$ Zhytomyr Ivan Franko State University,

40, Velyka Berdychivska str., 10008 Zhytomyr, Ukraine

*E-mail: kudryk@isp.kiev.ua

\begin{abstract}
The method of processing the data of electrophysical investigations of ohmic contacts has been developed. It allows obtaining more accurate results of measuring the contact resistance and additional information by analyzing the statistical and spatial distribution of input data. To test the method, the $\mathrm{Au}-\mathrm{Ge}-\mathrm{TiB}_{2}-\mathrm{Au}$ contact to $n-n^{+}-\mathrm{GaAs}$ was used. The analysis of frequency distribution for the total resistance, specific contact resistance and surface resistance of semiconductor has been carried out. The spatial distribution of these parameters has been analyzed. With taking the linear gradient of specific resistivity into account, the value of the contact resistance has been clarified. We have achieved reduction of half-width of the distribution by $14 \%$, that is, reduction of the error in determining the contact resistance. The method has been developed for correct analyzing the impacts of technological treatments and degradation processes and has been oriented on research purposes. Evaluation of the gradient distributions of the contact resistance and the resistance of semiconductor can be used to identify the defects in the technological processes of manufacturing devices.
\end{abstract}

Keywords: specific contact resistance, electrophysical diagnostic, ohmic contacts.

doi: https://doi.org/10.15407/spqeo22.01.11

PACS 73.40.Ns, 85.30.Kk

Manuscript received 08.01.19; revised version received 29.01.19; accepted for publication 20.02.19; published online 30.03.19.

\section{Introduction}

The development and improvement of ohmic contacts is one of the first steps in the developing technology of microelectronic devices based on a new material, also a step is often needed to optimize the existing technology. Therefore, the problem of correct study of parameters inherent to ohmic contacts in order to obtain the maximum information and trustworthiness is actual and timely. The existing set of methods for measuring the specific contact resistance, each of which has its limits of applicability, accuracy and specificity of use, was considered, for example, in [1-4], but in these works statistical aspect of measurements was not take into account. And in general, the experimenters, as a rule, do not enough take into account this aspect in processing the results of measurements.

\section{Method of data processing of ohmic contacts}

At the same time, the statistical measurements along the wafer may give not only a more accurate value of the contact resistance, but additional data related to the geometry of distributions of the calculated parameters, nature of errors and parameter gradients. In this work, the method of processing the data of measurements for ohmic contacts oriented on research purposes is proposed. In connection with the research direction, the visual control of processes that can occur in different ways depending on the technological conditions of formation of contacts, characteristics of the semiconductor surface and post-processing is widely used (see Fig. 1). 


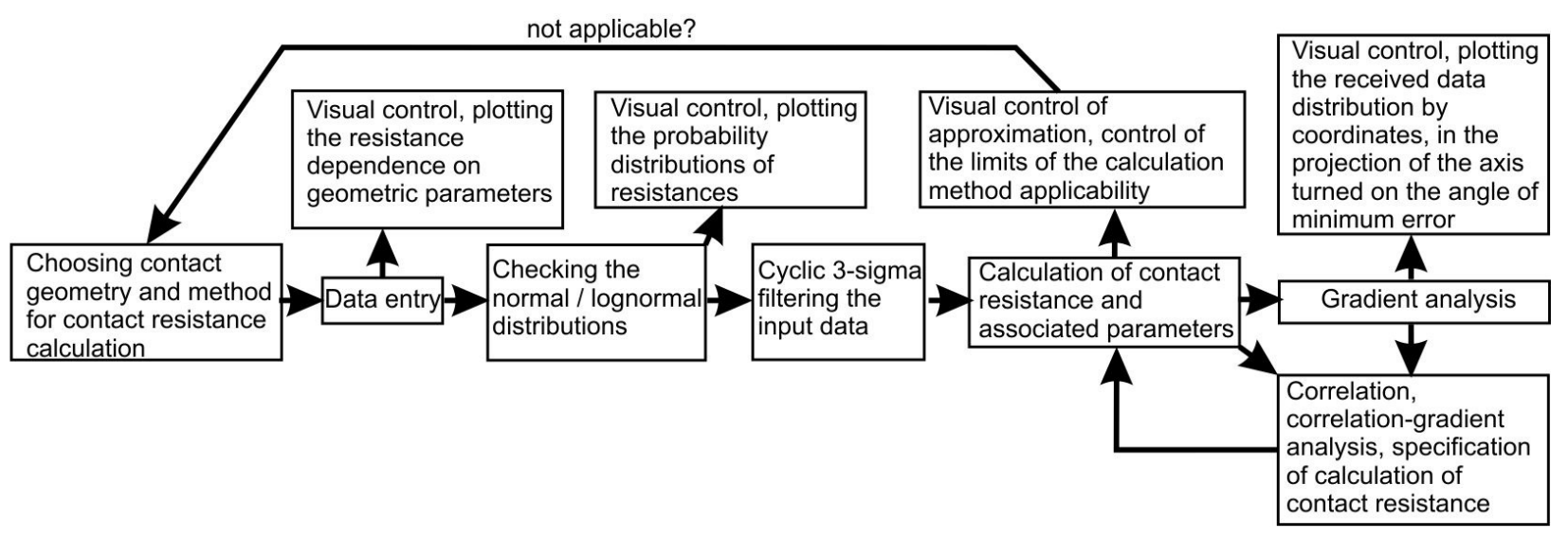

Fig. 1. Block-scheme of the method fок statistical processing the measurement results to calculate the contact resistance.

\section{Approbation of method}

To test the method, we used the contact of $\mathrm{Au}-\mathrm{Ge}-\mathrm{TiB}_{2}-$ Au to $n-n^{+}$with the concentration of carriers in the layer $n \sim 5 \cdot 10^{15} \mathrm{~cm}^{-3}$. The metallization was deposited using the magnetron method with the thicknesses of layers: $\mathrm{Au}$ $-1800 \AA$; $\mathrm{Ge}-300 \AA ; \mathrm{TiB}_{2}-1000 \AA ; \mathrm{Au}-2000 \AA$. After deposition of metal by using photolithography we form contact structures. Measuring the specific contact resistance was carried out using the method CTLM (circular transmission line model) (Fig. 2, insert). The temperature of rapid thermal annealing was $480{ }^{\circ} \mathrm{C}$.

\subsection{Analysis of small sample size}

The first step in studying the contact resistance, which should be made before photolithography, is to choose a measurement method or several alternative methods. The critical point of many numerical methods is checking on

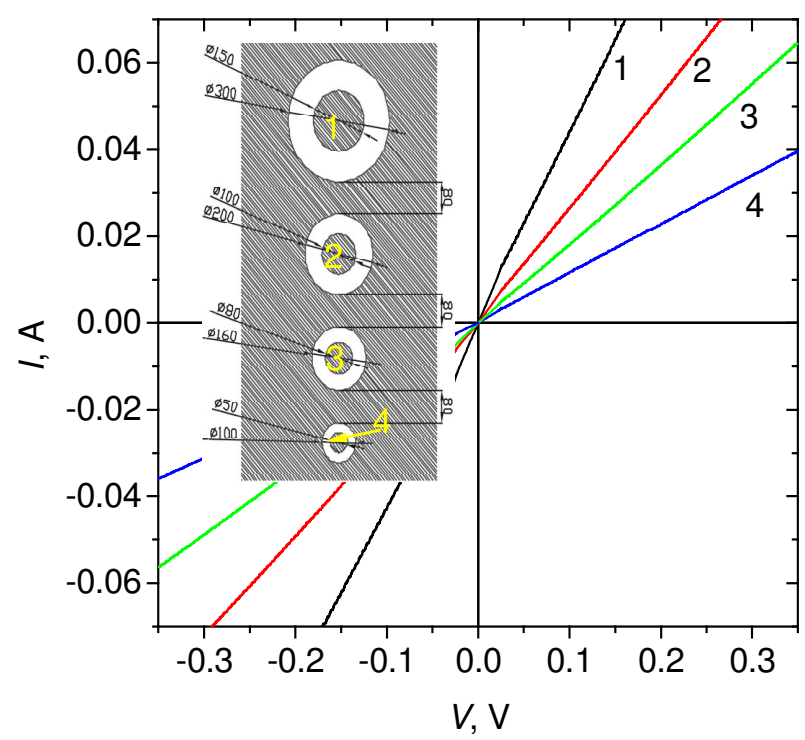

Fig. 2. Current-voltage characteristics of the contacts of one template. In the insert - a template of radial transmission line model (CTLM). the verge of applicability. Since the measurement of contacts of a large sample size is rather resourceintensive, the second step is the study of a small sample (up to 10 samples) that can be processed under a simplified scheme and is necessary for timely detection of ohmic contact parameters exceeding bounds of the study using this method and the need for a change in the measurement method. It is important to study the currentvoltage characteristics $(I-V)$ of contacts of the small sample size (Fig. 2). Formally, only the linearity and symmetry of $I-V$ give us the right to use the resistance to characterize the contact. Usually, the research of the contact resistance contains the dependence of the resistance on the geometric factor [1-4].

One of the criteria for the applicability of methods for calculating the contact resistance is good approximation of the experimentally obtained points with the theoretical curve (Fig. 3). In addition, for TLM methods the relationship between contact length and transfer length is often critical, for the vertical - the relationship between the diameter and thickness of the wafer.

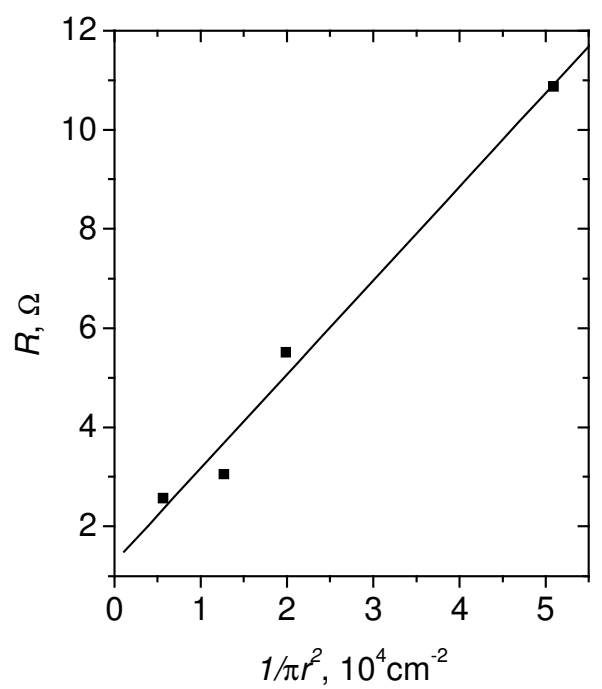

Fig. 3. Dependence of the resistance on the inverse contact area. 


\subsection{Analysis of large sample size}

If the results of a small sample size show the applicability of the method of measuring the contact resistance, then the next step is to study a large sample size of contact resistance. The distribution of resistance parameters will not necessarily be normal. It is even more likely that a normal distribution will be obtained only for the logarithm of resistance (lognormal distribution) [4], which should be taken into account when 3-sigma filtering the input data is used. Accordingly, the next step is to check the distributions for normality.

\subsubsection{Verification of distributions for normality and 3-sigma filtering}

The Shapiro-Wilk method is used to verify normality, which shows better results than other similar tests [5]. Fig. 4 shows the distribution histograms of the total resistance of the contacts with the diameters 50,80, 100 and $150 \mu \mathrm{m}$. It is evident that in the half-logarithmic coordinates, the distribution is closer to normal, which is confirmed by the Shapiro-Wilk test. It is especially noticeable when comparing Figs. $4 \mathrm{~g}$ and $4 \mathrm{~h}$, from this it follows that the distribution should be described by the lognormal dependence. Some deviation of this distribution from the lognormal dependence can be caused by the presence of two peaks of the distribution of resistance (see Fig. 4b, 4d), one of which may be related with the distribution of the contact resistance, another with the influence of the scatter of the semiconductor parameters and other factors unrelated with the contact resistance. That is why the first peak that is significant at small contact diameters (50 and $80 \mu \mathrm{m})$ becomes practically invisible on the contacts of larger diameter, in accordance with the reduction of contribution of the contact resistance in the total resistance of the contacts.

Only the knowledge that the distribution is close to lognormal gives grounds to carry out 3 -sigma filtering the input data, that is, to discard several points that are not part of the general distribution and are probably artifacts associated with the defectness of the semiconductor, setting before deposition of contact, lithography and more.

After filtering, we improve the values of median, mode and average values and a second 3-sigma filtration is performed until a point is rejected. Only after filtering the input data, we can go to the calculation of the specific contact resistance.

\subsubsection{Calculation of contact resistance and associated parameters}

In our case, in the method of calculating the contact resistance we used a template containing a set of contacts with a constant ratio of internal and external radii $r_{1} / r_{2}$ (see Fig. 2, the insert). For a single contact, the total resistancence can be written as in [6]:

$$
R=\frac{R_{s}}{2 \pi} \ln \left(\frac{r_{2}}{r_{1}}\right)+\frac{R_{s}}{2 \pi} \frac{1}{\alpha r_{1}} \frac{J_{0}\left(\alpha r_{1}\right)}{J_{1}\left(\alpha r_{1}\right)}
$$

where $r_{1}$ and $r_{2}$ are internal and external radii of the corresponding contacts; $J_{0}, J_{1}-$ modified Bessel functions of the first kind; $\alpha \equiv 1 / L_{t}$ is the inverse length of the transfer, $L_{t} \equiv \sqrt{\frac{\rho_{c}}{R_{S}}}, R_{S}$ is the specific surface resistance of the surface layer of the semiconductor; $\rho_{c}-$ specific contact resistance.

In the case where $L_{t}>>r_{1}$, the equation (1) can be simplified as follows:

$$
R=R_{s} C+\frac{\rho_{c}}{\pi r_{1}^{2}},
$$

where $C \equiv \frac{1}{2 \pi} \ln \left(\frac{r_{2}}{r_{1}}\right)$ is a constant. In other words, the dependence of the total resistance on the inverse contact area is linear with the slope coefficient equal to the specific contact resistance, as we observe in Fig. 3 for one of the templates. From each template on the plate, the specific contact resistance was calculated and a histogram of the frequency distribution of the specific contact resistance was plotted in linear and semilogarithmic coordinates (Fig. 5). It should be noted that only in semi-logarithmic coordinates the distribution passes the test Shapiro-Wilk for normality (Fig. 5b), which gives us the right to continue consideration of the distribution as lognormal.

Mathematical expectation calculated on the basis of approximation of distribution by the Gauss curve in Fig. $5 \mathrm{~b}$ is $1.52 \cdot 10^{-4} \mathrm{Ohm} \cdot \mathrm{cm}^{2}$. The average value is $1.60 \cdot 10^{-4} \mathrm{Ohm} \cdot \mathrm{cm}^{2}$, the median equals $1.52 \cdot 10^{-4} \mathrm{Ohm} \cdot \mathrm{cm}^{2}$, the mode is $1.60 \cdot 10^{-4} \mathrm{Ohm} \cdot \mathrm{cm}^{2}$.

\subsubsection{Correlation analysis}

Due to the scatter of the ohmic contact parameters, an error in the determination of the contact resistance may occur, which, in accordance with (2), when the contact resistance is exceeded, simultaneously leads to lowering the specific surface resistance of the semiconductor, and vice versa. That is, the error of this kind will lead to a negative correlation between $\rho_{c}$ and $R_{s}$. On the other hand, the correlation between $\rho_{c}$ and $R_{s}$ can be of physical nature, that is, changes in both parameters may be related to the variations in the concentration of the doping admixture in the film, which will lead to a positive correlation between the parameters. It is known that with a negative correlation, the replacement of $R_{s}$, determined from a specific template as the average value on the plate, can reduce the error in determination of $\rho_{c}$ [7].

In our case, a highly blurred negative correlation is observed (Fig. 6). Let re-calculate $\rho_{c}$, replacing $R_{s} C$ 

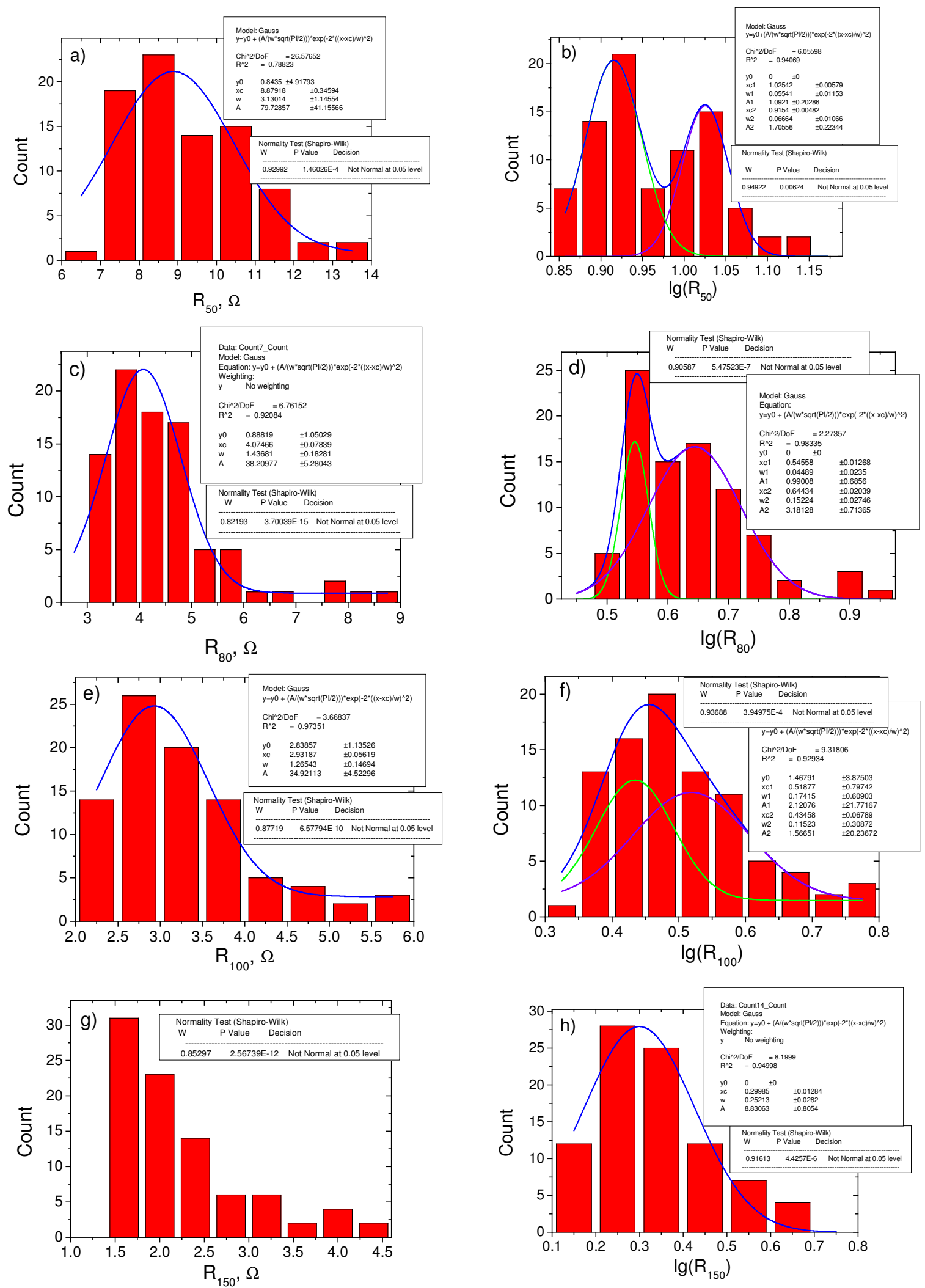

Fig. 4. Total contact resistance histograms for contacts $d=50 \mu \mathrm{m}(a, b), d=80 \mu \mathrm{m}(c, d), d=100 \mu \mathrm{m}(e, f)$, and $d=150 \mu \mathrm{m}(g, h)$ in linear $(a, c, e, g)$ and semi-logarithmic $(b, d, f, h)$ coordinates. 

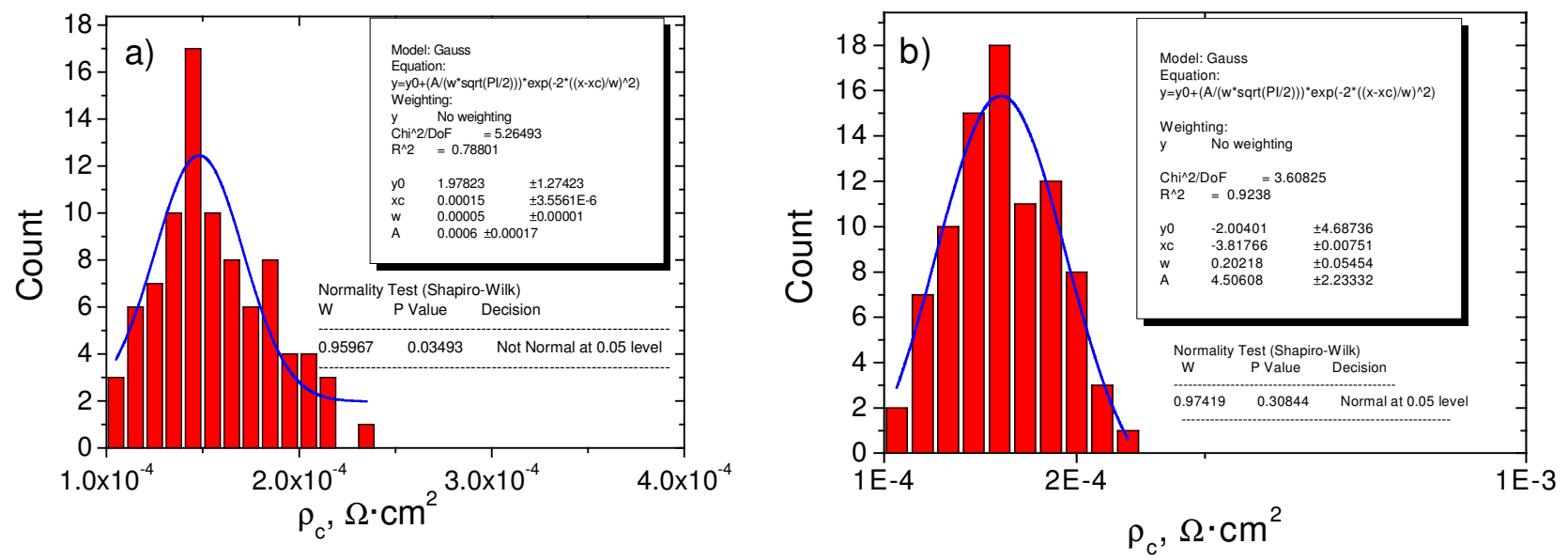

Fig. 5. Histograms of frequency distributions of specific contact resistance in linear (a) and semi-logarithmic (b) coordinates.

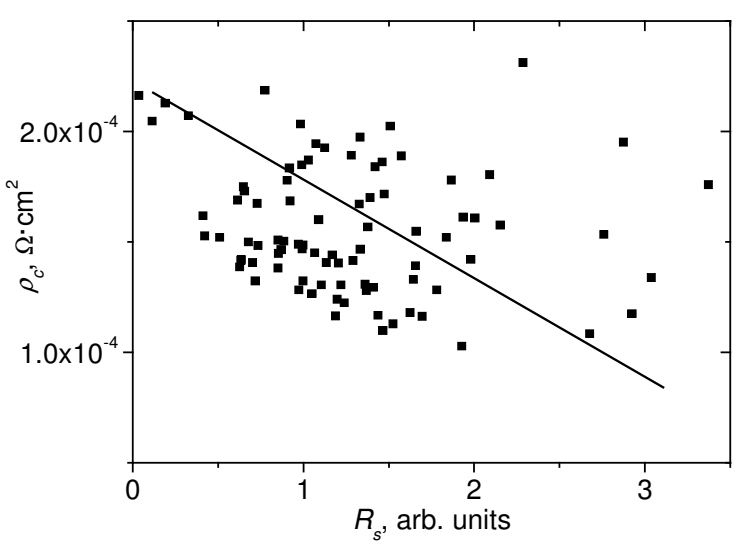

Fig. 6. Relationship between the calculated parameters $\rho_{c}$ and $R_{\mathrm{s}}$.

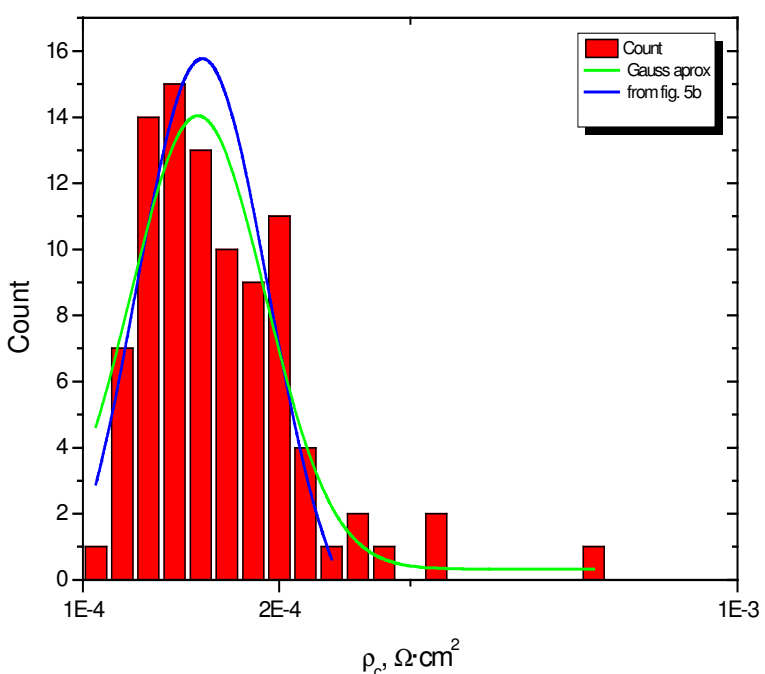

Fig. 7. Histogram of the frequency distribution of the specific contact resistance with the replacement $R_{s} C$ by $\overline{R_{s}} C$.

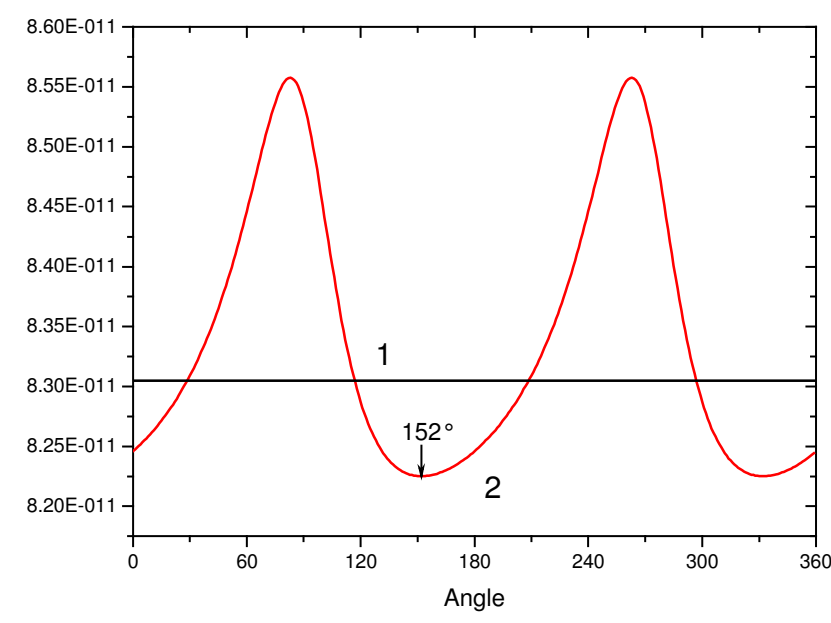

Fig. 8. Error of approximation of the dependence inherent to the specific contact resistance on the coordinate projection: 1 - radial distribution with the center defined from Fig. 9, and 2 - linear distribution depending on the angle of rotation.

determined for the specific template, on the mathematical expectation of a sample across the whole plate $\overline{R_{S}} C$, found on the basis of the frequency distribution of the specific surface resistance. We find the contact resistance for each template as the average weighted value $\rho_{c}^{*}$ found from the formula (2) for each of the template contact with weighted coefficients $\vartheta_{i} \equiv\left(\overline{R_{s}} C \pi r^{2} / \overline{\rho_{c}}+1\right)^{-4}$, proportional to the contribution of error of the total resistance to the contact resistance:

$$
\rho_{c}^{*}=\frac{\sum_{i=1}^{n} \vartheta_{i} \rho_{c i}}{n \sum_{i=1}^{n} \vartheta_{i}} .
$$




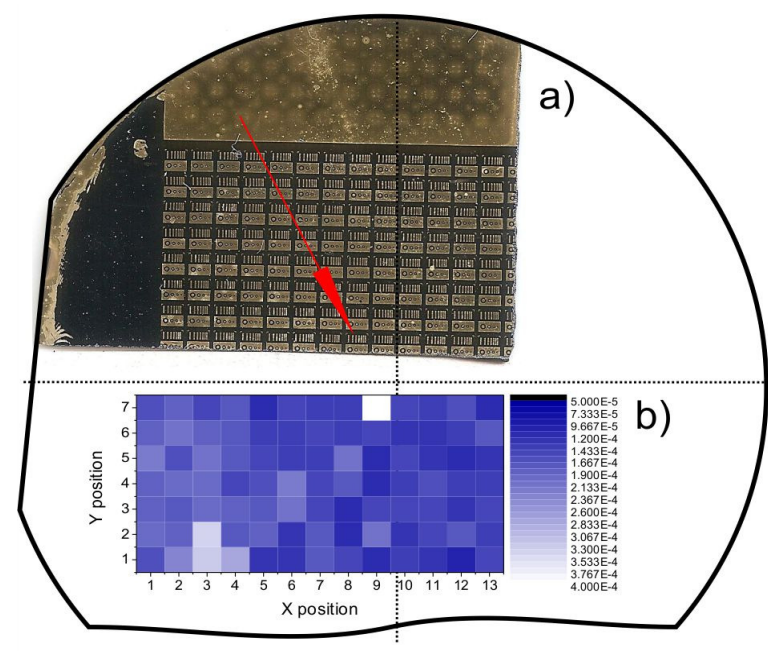

Fig. 9. Distribution of the contact resistance on the area of the plate (lighter colour - worse). The center of the radial distribution is marked with a dot. The red arrow indicates the optimal angle of the linear gradient from Fig. 8.

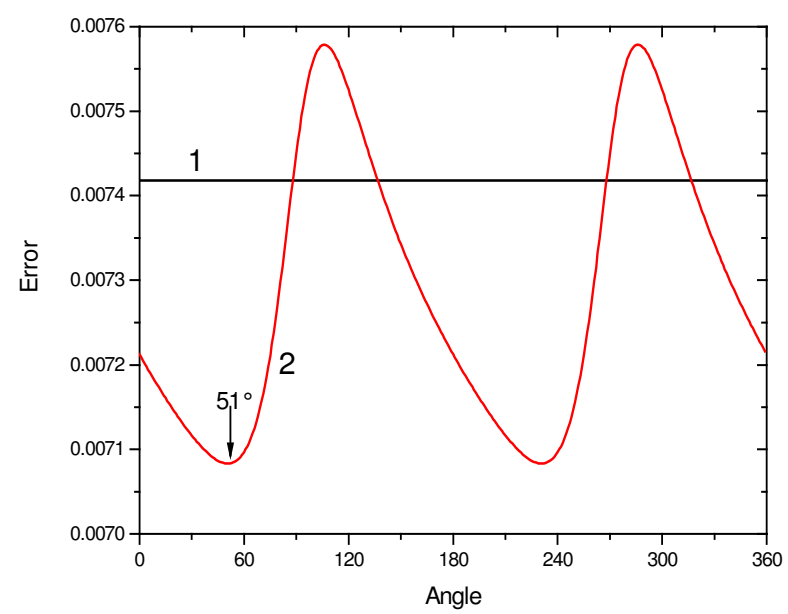

Fig. 10. Error of approximation of the dependence inherent to the specific resistivity of semiconductor on the coordinate projection: 1 - radial distribution with the center defined from Fig. 9 and 2 - linear distribution depending on the angle of rotation.

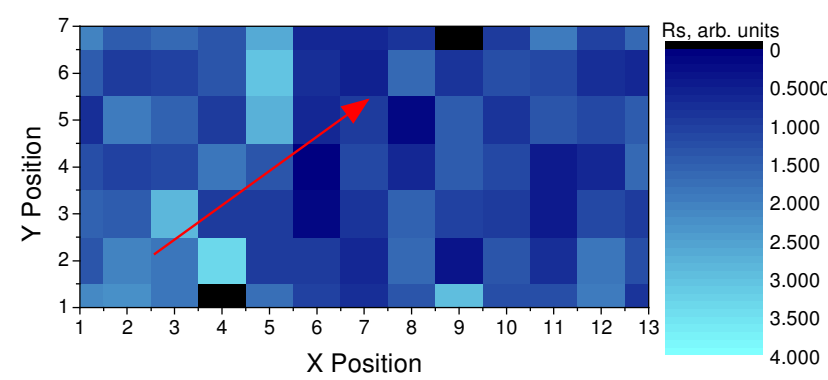

Fig. 11. Distribution of the specific resistivity of semiconductor on the area of the plate (lighter colour - worse). The center of the radial distribution is marked with a dot. The red arrow indicates the optimal angle of the linear gradient from Fig. 10.
In this case, this replacement does not improve the scatter of values of the calculated specific resistance (Fig. 7), which can testify to the simultaneous action of several factors of errors, in particular, the influence of the spatial distribution of semiconductor specific resistivity, which we will examine in the next paragraph.

\subsubsection{Gradient analysis}

Spatial heterogeneity can also contribute to the value of both the specific contact resistance and specific resistivity of the semiconductor. To study the presence of spatial distribution, two conditions were tested, namely: the presence of a linear gradient of the distribution of the contact resistance and presence of a radial distribution of the contact resistance with the center located in the center of the plate. The physical preconditions for the appearance of linear gradients are related with the technological processing of a part of the sample, in particular, the chemical processing of the surface and the rapid thermal annealing. The radial distribution can occur as a result of the transfer of growth inhomogeneities of the plate to the epitaxial film, which will affect the properties of the ohmic contact.

From Fig. 8, we see that the linear gradient of the distribution inherent to the specific resistance is more likely to be realized. Visually, we may observe it in Fig. 9b, as the color gradient from the lighter in the upper left corner to the darker in the lower right corner of the matrix. Similar graphs can be constructed for the specific resistance of the semiconductor (Figs. 10 and 11). The angle to the gradient of the semiconductor specific resistivity does not coincide with that for the contact resistance, which is understandable, since otherwise there would be a clear correlation between $\rho_{c}$ and $R_{s}$ with a connection through the geometric factor.

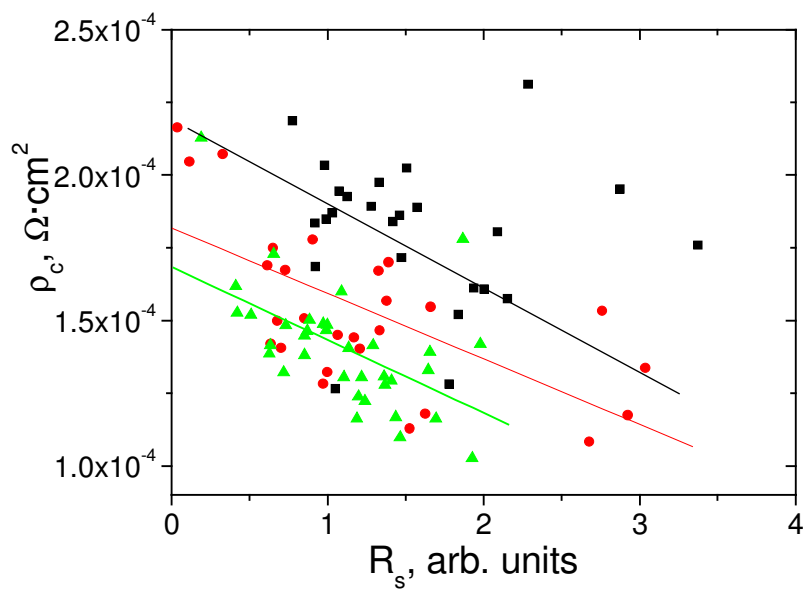

Fig. 12. Dependence between the calculated parameters $\rho_{c}$ and $R_{s}$ is separated into three identical groups of contacts by placement on the plate with the optimal angle of the linear gradient. 


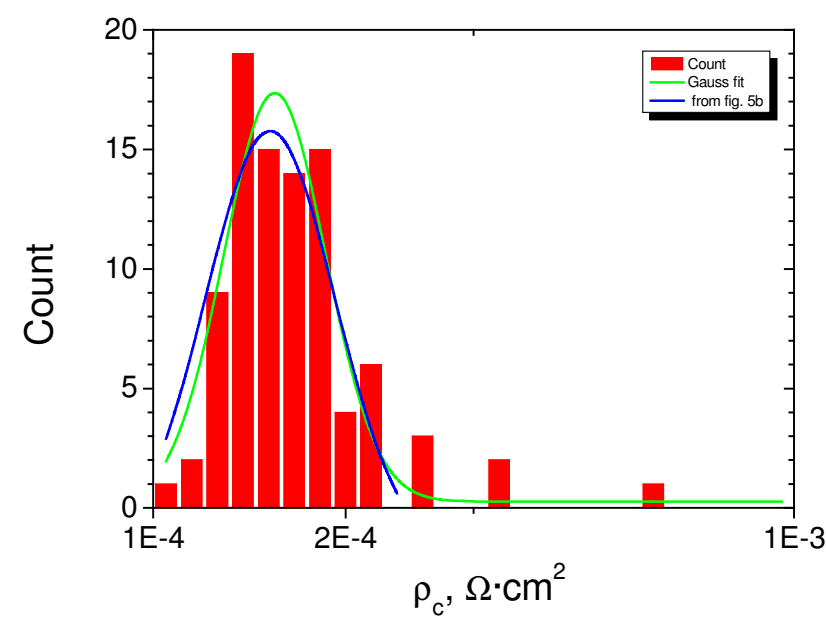

Fig. 13. Refined histogram of the frequency distribution inherent to the specific resistance of the contact.

More obviously, the same can be seen in Fig. 12, where the set of experimental points is separated by three identical subsets with their placement along the axis corresponding to the optimal gradient (Fig. 9), the influence of the geometric factor on the blurring of dependence between the parameters $\rho_{c}$ and $R_{s}$ is clearly seen. Accordingly, to improve the value of the contact resistance, we will replace $R_{s}$ with the linear gradient of resistivity and re-calculate the value of the contact resistance. As can be seen from Fig. 13, this substitution results in a significant reduction in the half-width of the distribution by $14 \%$, that is, enables to reduce the error in determining the contact resistance.

In our case, the ohmic contacts are formed at a sufficiently high annealing temperature [2, 8-11], so the scatter of the values of the contact resistance is minimal, but even in this situation, the method allows to obtain additional information on the sources of the scatter in the contact resistance parameters, which may be useful in improvement the technological processes of forming contacts or quality control of experimental production.

\section{Conclusions}

The method of processing data of electrophysical investigations of ohmic contacts has been developed, which allows obtaining more accurate results of measuring the contact resistance and obtaining additional information by analyzing the statistical and spatial distributions of input data. It has been shown that the distribution of the specific contact resistance, at least in this case, is described by the lognormal dependence, which is confirmed by the Shapiro-Wilk test. The method has been developed for the correct analysis of the impacts of technological treatments and degradation processes and has been oriented on research purposes. Evaluation of the gradient distributions of the contact resistance and resistance of semiconductor can be used to identify limitation in the technological processes of production of devices.

\section{References}

1. Sidhant Grover. Effect of Transmission Line Measurement (TLM) Geometry on Specific Contact Resistivity Determination. A thesis for the degree of Master of Science in Materials Science and Engineering in the School of Chemistry and Materials Science, College of Science Rochester Institute of Technology, December 2016.

2. Holland A.S., Reeves G.K. New challenges to the modelling and electrical characterization of ohmic contacts for ULSI devices. Microelectronics Reliability. 2000. 40, No 6. P. 965-971; doi: 10.1109/ICMEL.2000.838732.

3. Berger H.H., Holland A.S., Reeves G.K. Contact resistance and contact resistivity. J. Electrochem. Soc. 1972. 119, No 4. P. 507-514. doi: 10.1149/1.2404240.

4. Sheremet V.N. Metrological aspects of measuring resistance of ohmic contacts. Radioelectronics and Communications Systems. 2010. 53, Issue 3. P. 119-128; https://doi.org/10.3103/S0735272710030015.

5. Razali N.M., Wah Y.B. Power comparisons of Shapiro-Wilk, Kolmogorov-Smirnov, Lilliefors and Anderson-Darling tests. Journal of Statistical Modeling and Analytics. 2011. 2, No 1, P. 21-33.

6. Reeves G.K. Specific contact resistance using a circular transmission line model. Solid-State Electronics. 1980. 23. No 5. P. 487-490. https://doi.org/10.1016/0038-1101(80)90086-6.

7. Basanets V.V., Slepokurov V.S., Shynkarenko V.V., Kudryk Ya.Ya, Kudryk R.Ya, Konakova R.V., Kovtonuk V.M. Studying the resistivity of ohmic contacts $\mathrm{Au}-\mathrm{Ti}-\mathrm{Pd}-n-\mathrm{Si}$ for avalanche transit-time diodes. Tekhnologiya i konstruirovaniye $v$ elektronnoy apparature. 2015. №1. C. 33-37 (in Russian); doi: 10.15222/TKEA2015.1.33.

8. Belyaev A.E., Boltovets N.S., Kapitanchuk L.M. et al. The features of temperature dependence of contact resistivity of $\mathrm{Au}-\mathrm{Ti}-\mathrm{Pd}_{2} \mathrm{Si}-p^{+}-\mathrm{Si}$ ohmic contacts. Semiconductor Physics, Quantum Electronics \& Optoelectronics. 2010. 13, No 1. P. 8-11.

9. Sachenko A.V., Belyaev A.E., Boltovets N.S. et al. On a feature of temperature dependence of contact resistivity for ohmic contacts to $n$-Si with an $n^{+}-n$ doping step. Semiconductor Physics, Quantum Electronics \& Optoelectronics. 2014. 17, No 1. P. $1-6$.

10. Belyaev A.E., Pilipenko V.A., Anischik V.M. et al. Role of dislocations in formation of ohmic contacts to heavily doped $n$-Si. Semiconductor Physics, Quantum Electronics \& Optoelectronics. 2013. 16, No 2. P. 99-110.

11. Shepela A. The specific contact resistance of $\mathrm{Pd}_{2} \mathrm{Si}$ contacts on $n$ - and $p$-Si. Solid-State Electronics. 1973. 16, Issue 4. P. 477-481. https://doi.org/10.1016/0038-1101(73)90185-8. 


\section{Authors and CV}

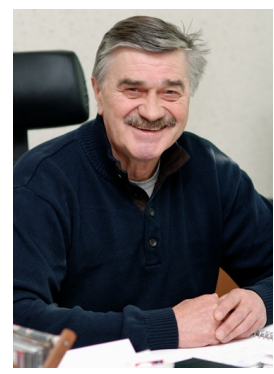

A.E. Belyaev, Director of V. Lashkaryov Institute of Semiconductor Physics, Academician of NAS of Ukraine, Professor, Doctor of Sciences. The area of his scientific interests includes electrical and galvanomagnetic properties of semiconductors.

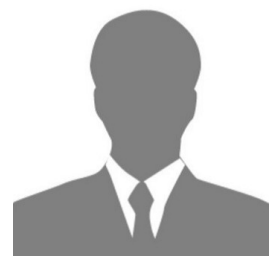

M.S. Boltovets, Head of Department at the State Enterprise "Research Institute "Orion". The area of his scientific interests includes IMPATT and Gunn diode technology, reliability of semiconductor devices.

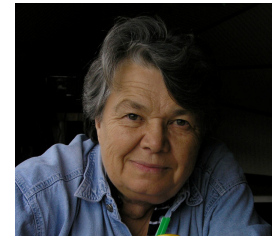

R.V. Konakova, Professor, Doctor of Sciences, Head of Laboratory for physical and technological problems of solid-state microwave electronics at the V. Lashkaryov Institute of Semiconductor Physics. The area of her scientific interests includes solid state physics, transport properties in metal-semiconductor contacts, reliability of semiconductor devices.

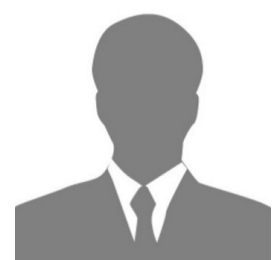

V.M. Kovtonjuk, Researcher at the State Enterprise "Research Institute "Orion". The area of his scientific interests includes IMPATT and Gunn diode technology, generator efficiency.

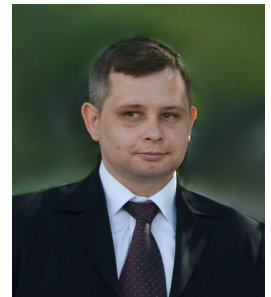

Ya.Ya. Kudryk, Senior researcher at the V. Lashkaryov Institute of Semiconductor Physics. The area of his scientific interests includes solid state physics, transport properties in metal-semiconductor contacts to $\mathrm{SiC}$, $\mathrm{GaN}, \mathrm{GaP}, \mathrm{InP}$.

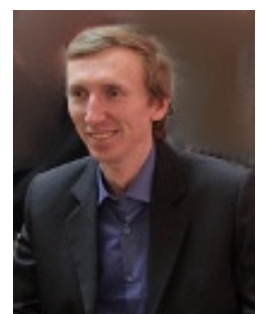

V.V. Shynkarenko, Senior researcher of V. Lashkaryov Institute of Semiconductor Physics. The area of his scientific interests includes solid state physics, thin film physics, highfrequency devices.

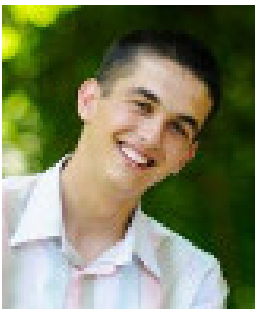

M.M. Dub The area of his scientific interests includes solid state physics, transport properties in metalsemiconductor contacts to diamond.

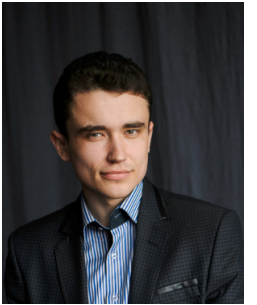

P.O. Saj The area of his scientific interests includes solid state physics, transport properties in metalsemiconductor contacts to $\mathrm{InN}$.

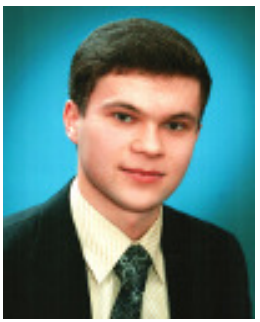

S.V. Novitskii, Senior Lecturer of Zhytomyr Ivan Franko State University. The area of his scientific interests includes solid state physics, transport properties in metalsemiconductor contacts to InP. 